\title{
Embarazadas con hisopado positivo para SARS-CoV-2: presentación clínica y evolución de la dupla madre- recién nacido de una población hospitalaria
}

\section{Pregnant women with a positive swab for SARS-CoV-2: clinical presentation and evolution of the mother-newborn pair in a hospital population}

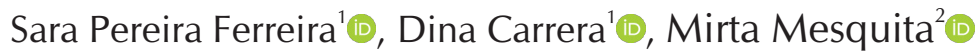 \\ ${ }^{1}$ Instituto de Previsión Social, Hospital Central. Asunción, Paraguay. \\ ${ }^{2}$ Hospital General Pediátrico Niños de Acosta Nu, Departamento de Docencia e Investigación. San \\ Lorenzo, Paraguay.
}

\section{RESUMEN}

Introducción: Durante la pandemia, todas las mujeres embarazadas fueron sometidas a hisopado nasofaríngeo para SARS-CoV-2. Objetivo: Describir las características clínicas y evolución de las embarazadas con estudio de PCR positivo para SARS-CoV-2 y sus recién nacidos en una población hospitalaria. Materiales y Métodos: Estudio observacional ambispectivo de seguimiento de una cohorte, en la maternidad del Instituto de Previsión Social entre marzo 01 de 2020 a mayo 31 de 2021. Participaron embarazadas del tercer trimestre ingresadas para parto, con resultado positivo para SARS-CoV-2. Variables: edad, síntomas, complicaciones del embarazo, clasificación de COVID-19, ingreso a terapia intensiva, antropometría neonatal, seguimiento de la dupla madreneonato hasta los 7 días post parto. Datos analizados en SPSS utilizando estadísticas descriptivas. Protocolo aprobado por el comité de ética de la investigación. Resultados: Ingresaron 136 embarazadas y 139 neonatos (3 gemelares). El 78,6\% sintomáticas, 8,1\% presentó formas graves. El 10,3\% presentó preeclampsia, el 10,3\% ingresó a terapia intensiva y el 2,2\% falleció. El 86,4\% nació por cesárea, hubo 2 mortinatos. El 29,2\% fue-pretérmino, el $18,2 \%$ de bajo peso de nacimiento, $24,8 \%$ fue-hospitalizado Fallecieron 3/137 antes de los 7 días. El hisopado neonatal fue positivo en 3. El $78 \%$ se alimentó con pecho materno. Conclusión: El 15,5\% de embarazadas positivas

\section{ABSTRACT}

Introduction: During the pandemic, all pregnant women underwent a nasopharyngeal swab for SARS-CoV-2. Objective: To describe the clinical characteristics and evolution of pregnant women with a positive PCR study for SARS-CoV-2 and their newborns in a hospital population. Materials and methods: This was an ambispective observational, cohort follow-up study, in the maternity ward of the Social Security Institute Hospital between March 01, 2020 to May 31, 2021. Third trimester pregnant women admitted for delivery, with a positive result for SARS-CoV-2, were included. Variables: age, symptoms, pregnancy complications, COVID-19 classification, admission to intensive care, neonatal anthropometry, follow-up of the mother-neonate pair up to 7 days postpartum. The data was analyzed in SPSS using descriptive statistics. The protocol was approved by the research ethics committee. Results: 136 pregnant women and 139 newborns ( 3 twins) were admitted. $78.6 \%$ were symptomatic, $8.1 \%$ had severe forms. $10.3 \%$ presented pre-eclampsia, $10.3 \%$ entered intensive care and $2.2 \%$ died. $86.4 \%$ were born by cesarean section, there were 2 stillbirths. $29.2 \%$ were preterm, $18.2 \%$ had low birth weight, $24.8 \%$ were hospitalized. $3 / 137$ died before 7 days of age. The neonatal swab was positive in $3.78 \%$ were breastfed. Conclusions: $15.5 \%$ of positive pregnant women were admitted with moderate to severe forms of

Correspondencia: Mirta Mesquita Correo: mirtanmr@gmail.com Conflicto de interés: los autores declaran no poseer conflicto de interés Fuente de financiamiento: El estudio no conto con financiación externa Recibido:3/11/2021 Aceptado: 19/11/2021 DOI: https://doi.org/10.31698/ped.48032021004

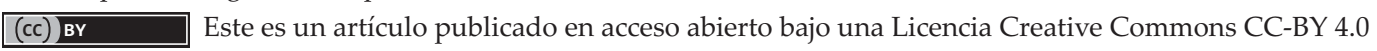


ingresaron con formas moderada a grave, de COVID 19, el 10,3\% ingresó a UTI y 3 fallecieron. El porcentaje de cesárea, prematuridad y hospitalización neonatal fue muy elevado. Hubo 2 mortinatos y el 2,9\% de los nacidos vivos fallecieron. El hisopado neonatal fue positivo en el 2,2\%.

Palabras claves: COVID-19, embarazo, neonatos, morbilidad, mortalidad

\section{INTRODUCCIÓN}

En diciembre del 2019, se registró un brote de infección respiratoria en la provincia de Wuhan China, producido por un nuevo coronavirus, relacionado al SARS-CoV. La OMS denominó a la enfermedad COVID-19, al nuevo coronavirus SARS-CoV-2, y meses después se declaró Pandemia $^{(1)}$. Considerando la rápida propagación de la enfermedad en China y el incremento de nuevos casos en países de la región, en Europa y los EEUU, se planteó la transmisión de persona a persona a través de gotas provenientes de la tos, estornudos o por contacto. Aunque la mayor parte de los casos fueron cuadros clínicos leves, un grupo de personas, adultas mayores y con enfermedad de base presentaron morbimortalidad elevada ${ }^{(2)}$.

Las mujeres embarazadas y los neonatos constituyen un grupo vulnerable a infecciones durante epidemias o pandemias. En un metaanálisis multinacional que incluyó 790 mujeres embarazadas con COVID-19 Dubey y cols, encontraron eventos adversos perinatales en el $27 \%$, pero con cifras muy heterogéneas dependiendo del país donde se realizó el estudio. Sin embargo, en otro metaanálisis Figueiro.Filho y cols, analizaron las complicaciones y mortalidad de más de 10 mil embarazadas con COVID-19, y no encontraron diferencias con la población general ${ }^{(3)}$. Sin duda los estudios analizados eran muy heterogéneos a pesar de la metodología utilizada. Existen diferencias en las diferentes regiones geográficas, en la disponibilidad de diagnóstico y sobre todo en la terapéutica ${ }^{(4)}$.Un estudio latinoamericano que incluyó 86 embarazadas COVID-19 positivas de 7 países de la red de la Sociedad Iberoamericana de Neonatología,
COVID 19, 10.3\% were admitted to the ICU and 3 died. The percentage of caesarean section, prematurity and neonatal hospitalization was very high. There were 2 stillbirths and $2.9 \%$ of live births died. The neonatal swab was positive in $2.2 \%$.

Key words: COVID-19, pregnancy, neonates, morbidity, mortality.

no encontró efectos adversos perinatales graves, y sí un porcentaje elevado de separación de la diada madre-neonato ${ }^{(5)}$.

Mas recientemente un estudio de cohorte que incluyó a más de 2 mil embarazadas con y sin COVID-19, procedentes de 18 países, de los cuales 2 eran latinoamericanos, encontró asociación del COVID-19 con preeclampsia, parto prematuro y mayor mortalidad maternal. Los neonatos de madres positivas, presentaron mayor morbimortalidad ${ }^{(6)}$.

La transmisión vertical del SARS-CoV-2 se ha reportado con escasa evidencia. La mayoría fueron catalogadas como posibles infecciones connatales, considerando que no se posee evidencia de la presencia del virus en el feto ${ }^{(7)}$.

Durante la pandemia, por protocolo todas las mujeres embarazadas que ingresan para el parto $u$ otra razón son sometidas a hisopado nasofaríngeo paraSARS-CoV-2.

En este contexto el objetivo del presente estudio fue describir las características clínicas y evolución de las embarazadas con estudio de PCR positivo para SARS-CoV-2 y sus recién nacidos en una población hospitalaria.

\section{MATERIALES Y MÉTODOS}

\section{Diseño del estudio y población}

Estudio observacional descriptivo ambispectivo de seguimiento de una cohorte, llevado a cabo en la maternidad del Hospital Central del Instituto de 
Previsión Social, en el periodo comprendido entre marzo 01 de 2020 y mayo 31 de 2021. Por un muestreo no probabilístico de casos consecutivos, fueron incluidas embarazadas del tercer trimestre, ingresadas para el parto, sometidas al hisopado nasofaríngeo para SARS-CoV-2 y cuyo resultado fue positivo.

\section{Procesamiento de la muestra}

Las muestras fueron tomadas por hisopado nasofaríngeo, y llevadas al laboratorio de biología molecular, en un medio de transporte (Vircell@) para su procesamiento. Se procedió a la extracción del ARN viral en forma manual en columna utilizando el kit Promega ${ }^{\circledR}$ USA y se realizó la reacción en cadena de la polimerasa en tiempo real (PCR) utilizando el termociclador CFX96 (BioRad), USA. El reactivo de detección del SARS-CoV-2 utilizado fue: Itaq Universal Probes One-Step KIT BioRad. Los genes virales amplificados fueron: los genes $\mathrm{E}$ y $\mathrm{N}$.

\section{Medición de las variables}

Por medio de un cuestionario elaborado para el efecto, los datos fueron recogidos a partir de la historia clínica materna, y corroborados a través de entrevista telefónica con las participantes. Las variables estudiadas fueron edad, procedencia, la presencia de síntomas atribuibles al SARS-CoV-2, comorbilidades, clasificación de la gravedad del COVID-19 de acuerdo al Protocolo para COVID-19 elaborado por el Ministerio de Salud Pública y Bienestar Social (Guía para el manejo de mujeres embarazadas y eventos obstétricos $)^{(8)}$ e ingreso a la unidad de cuidados intensivos. Se consignó además las características del parto, presencia de complicaciones, datos del recién nacido (peso de nacimiento, edad gestacional, test de Apgar al minuto y a los 5 minutos), resultado del hisopado para SARS-CoV-2, alimentación, relacionamiento con las madres, necesidad de hospitalización, estado al alta y posterior evolución de los recién nacidos y las madres hasta el séptimo día del alta hospitalaria, por medio de contacto telefónico.

La edad gestacional se determinó por ecografía del primer trimestre y/o el test de Capurro al nacer.

\section{Análisis de los datos}

Los datos fueron analizados con el programa SPSS, utilizando estadísticas descriptivas. Las variables cuantitativas se presentan como medias con desvíos estándar o medianas con rangos intercuartílicos de acuerdo a su distribución, determinada por el Test de Kolmogorov-Smirnov. Las variables cualitativas se presentan como porcentajes.

\section{Aspectos éticos}

El protocolo fue aprobado por el comité de ética de investigación, con consentimiento informado verbal, realizado en el momento de la consulta telefónica con las participantes y/o personas autorizadas.

\section{RESULTADOS}

Durante el periodo de estudio fueron incluidas 136 mujeres embarazadas y 139 neonatos (3 embarazos gemelares).

La vía del parto fue cesárea en 86,8\% (118/136) y presentaron complicaciones del embarazo en el $14,7 \%$ (20/136). Los datos demográficos y los tipos de complicaciones se muestran en la Tabla1.

En relación a la forma clínica de presentación en el momento del diagnóstico, el 21,3\% (29/136) eran asintomáticas. En el grupo de embarazadas sintomáticas $(\mathrm{n}=107)$ predominó los síntomas de infección de vías aéreas superiores como congestión nasal y rinorrea. Otros síntomas, así como la clasificación de la gravedad del COVID-19 se encuentran en la tabla 2.

El 18,3\% (25/136) de las embarazadas presentó comorbilidad, (diabetes 9,5\% (13/136), Asma 2,2\% (3/136), hipotiroidismo 2,2\% (3/136), Obesidad 1,5\% $(2 / 136)$, sífilis $1,5 \%(2 / 136)$, artritis reumatoidea y adicción a heroína en el 0,7\% (1/136) respectivamente).

Analizando las características neonatales, hubieron 2 mortinatos, ambos fueron de pretérmino: el primero de sexo femenino, con peso de 2390 gramos y 35 semanas de edad gestacional por ecografía. El segundo de sexo masculino con peso de 1300 gramos y 30 semanas de edad gestacional por ecografía, hijo de una mujer con la forma grave de COVID-19 que también falleció.

Fueron analizadas las características de los 137 neonatos nacidos vivos. El 54,7\% (75/137) fueron de 
sexo femenino y el 29,2\% (40/137) fueron prematuros. Otros datos neonatales se muestran en la tabla 3.

Tabla 1. Características demográficas y perinatales de las mujeres embarazadas con hisopado para SARS-CoV-2 positivo. $\mathrm{N}=136$

\begin{tabular}{lr}
\hline $\begin{array}{l}\text { Edad materna (años) } \\
\text { Media DE }\end{array}$ & $29,2 \pm 5,9$ \\
\hline Edad gestacional *(semanas) & $37(36-39)$ \\
$\quad$ Mediana (P25 -P75) & $\mathbf{N} \mathbf{( \% )}$ \\
\hline Procedencia & $94(69,1)$ \\
$\quad$ Departamento central & $22(16,2)$ \\
Asunción & $20(14,7)$ \\
Otros departamentos & $118(86,8)$ \\
\hline Vía del parto & $18(13,2)$ \\
Cesárea & $20(14,7)$ \\
Vaginal & $14(10,3)$ \\
\hline Complicaciones del Embarazo & $2(1,5)$ \\
\hline Tipo de Complicaciones $(\mathbf{n}=\mathbf{2 0})$ & $3(2,2)$ \\
Preeclampsia & $1(0,7)$ \\
Infecciosa & \\
Colestasis del Embarazo & \\
Percretismo placentario & \\
\hline
\end{tabular}

*edad gestacional en el momento del hisopado de la madre.

Tabla 2. Manifestaciones Clínicas y Gravedad de COVID-19 en las mujeres embarazadas sintomáticas. $\mathrm{N}=107$

\begin{tabular}{lc}
\hline Síntomas & $\mathbf{N ~ ( \% )}$ \\
\hline De Vías Aéreas Superiores & $51(47,7)$ \\
Fiebre más dificultad respiratoria & $15(14,0)$ \\
Fiebre sin otros síntomas acompañantes & $13(12,1)$ \\
Fiebre más síntomas de VAS & $11(10,3)$ \\
Anosmia (más fiebre o síntomas de VAS) & $8(7,5)$ \\
Dificultad respiratoria & $7(6,5)$ \\
Insuficiencia respiratoria & $1(0,9)$ \\
Cefalea y cansancio & $1(0,9)$ \\
\hline Clasificación según gravedad $(\mathbf{N}=\mathbf{1 3 6})$ & \\
\hline Leve & $115(84,5)$ \\
Moderada & $10(7,4)$ \\
Grave & $11(8,1)$ \\
\hline
\end{tabular}

El 98,5\% (135/137) de los neonatos fue sometido a hisopado nasofaríngeo entre las 2 y las 144 horas de vida, con una mediana de 24 horas, (P25 =12h - P75= $24 \mathrm{~h})$. El 2,2\% (3/135) fue positivo. Se analizó la relación entre la edad en el momento de la toma de muestra y los resultados positivos y negativos de la
PCR para SARS-CoV-2. La mediana de la edad postnatal en que fueron tomadas las muestras en los positivos fue de 24 horas con un mínimo de 4 horas y un máximo de 96 horas. Mientras que la edad mediana de los negativos $(n=132)$ fue de 24 horas con un mínimo de 2 horas a máximo de 144 horas. Los 3 neonatos con resultados positivos fueron hijos de madres con COVID-19 leve y tuvieron buena evolución clínica. Analizando la evolución de la diada madre-neonato.

El 24,8\% (34/137) fueron hospitalizados, el 73\% (100/137) permanecieron con sus madres y solo el $2,2 \%$ (3/137) fueron separados. En el periodo perinatal $3 / 137$ fallecieron y 1 neonato dado de alta fue rehospitalizado. Después de la semana de vida 1 neonato falleció En cuanto a la evolución de las madres el 10,3\% requirieron terapia intensiva. Fallecieron el 2,2\% (3/136) dentro de la semana del post parto. Tabla 4

Tabla 3. Características de los neonatos, nacidos vivos, hijos de Madres con Hisopado Positivo para SARS-CoV-2. $\mathrm{N}=137$

\begin{tabular}{lc}
\hline $\begin{array}{l}\text { Peso de nacimiento } \\
\text { Media DE }\end{array}$ & $\begin{array}{c}\text { (Gramos) } \\
\text { Edad gestacional } \\
\text { Mediana (P25-P75) }\end{array}$ \\
\hline Apgar al minuto & (Semanas) \\
Mediana (P25-P75) & $37(36-39)$ \\
\hline Apgar a los 5 min. & $8(8-8)$ \\
Mediana (P25 - P75) & \\
\hline Sexo & $9(9-9)$ \\
Masculino & $\mathbf{N ~ ( \% )}$ \\
Femenino & $62(45,3)$ \\
\hline Clasificación: Peso/Edad gestacional & $75(54,7)$ \\
Adecuado & $120(87,6)$ \\
Grande & $10(7,3)$ \\
Pequeño & $7(5,1)$ \\
Prematuros & $40(29,2)$ \\
Bajo peso de nacimiento & $26(19,0)$ \\
\hline
\end{tabular}


Tabla 4. Evolución clínica materna y neonatal post parto, y a los $\geq 7$ días del alta hospitalaria.

\begin{tabular}{lrlr}
\hline $\begin{array}{l}\text { Evolución materna } \\
\text { post parto } \mathbf{n = 1 3 6}\end{array}$ & $\mathbf{N}(\%)$ & $\mathbf{l}$ Evolución neonatal \\
Ingreso a UTI & $14(10,3)$ & Hospitalizados & $34(24,8)$ \\
Sala maternidad & $122(89,7)$ & Alojamiento & $100(73,0)$ \\
& & Separación & $3(2,2)$ \\
\hline Evolución $<\mathbf{7}$ días & & Evolución post & \\
post parto $\mathbf{n = 1 3 6}$ & & alta $<$ 7d & \\
Alta & $122(89,7)$ & Fallecidos & $3(2,2)$ \\
UTI* & $11(8,0)$ & Re hospitalizados & $1(0,7)$ \\
Fallecida & $3(2,2)$ & Buen estado $133(97,1)$ \\
\hline Evolución $\geq 7$ días & & Estado a $\geq 7$ días \\
post parto $\mathbf{n}=\mathbf{1 3 3}$ & & $\mathbf{n}=\mathbf{1 3 4}$ & \\
Buen estado & $127(95,5)$ & Buen estado & $133(99,3)$ \\
Hospitalizadas & $6(4,5)$ & Fallecido & $1(0,7)$ \\
\hline
\end{tabular}

$\mathrm{UTI}=$ Unidad de terapia intensiva

El 78,1\% (107/137) se alimentó con pecho materno, el $8 \%(11 / 137)$ recibió alimentación mixta, formula y leche materna y el 13,9\% (19/137) tuvo ayuno las primeras horas en la unidad de cuidados intensivos neonatales.

Los diagnósticos de los neonatos hospitalizados fueron Prematurez 6/34, distress respiratorio 9/34, Sepsis neonatal precoz 5/34. Miocarditis 2/34, Asfixia perinatal 1/34 y Cardiopatis congénita 1/34.

De los 4 neonatos fallecidos 2 eran de pretérmino, poner coma y seguir de 28 semanas y 1220 g, 30 semanas y $1400 \mathrm{~g}$ de edad gestacional y peso de nacimiento respectivamente. Los otros 2 neonatos fallecidos eran de termino con asfixia perinatal severa y cardiopatía congénita respectivamente.

\section{DISCUSIÓN}

En el presente estudio la mayoría de las embarazadas con COVID-19, eran sintomáticas al ingreso hospitalario, y con afectación leve de la enfermedad. El porcentaje de casos graves fue bajo. Aunque uno de los primeros metaanálisis realizado en el 2020, sobre las características del COVID-19 y sus efectos en las embarazadas, reportaron buen pronóstico, y sin efectos adversos perinatales ${ }^{(5,9)}$, los realizados posteriormente, con mayor número de pacientes encontraron elevado porcentaje de efectos adversos, predominio de nacimiento por cesárea, $\mathrm{y}$ de prematuros e ingreso materno a la unidad de cuidados intensivos ${ }^{(10,11)}$. En la población de embarazadas del tercer trimestre, del presente estudio, fueron testadas para SARS-CoV-2 como parte del protocolo sanitario al ingresar al hospital. El elevado porcentaje de cesárea y neonatos de pretérmino hallados, son similares a lo reportado por Antoun y cols, en Inglaterra, quienes encontraron $84 \%$ de cesárea y $37 \%$ de partos pretérminos en embarazadas infectadas en el tercer trimestre del embarazo $^{(12)}$, y es mucho mayor que los datos nacionales del Ministerio de Salud Pública de Paraguay del año 2018, que muestra que el 50,9\% de los partos fueron por cesárea ${ }^{(13)}$. Los casos graves tuvieron elevada mortalidad, y solo una de las 3 mujeres fallecidas tenía comorbilidad. Algunos autores sostienen que la mayor morbimor-talidad de la COVID-19 en embarazadas podría estar ligado a los cambios fisiológicos producidos por el embarazo como mayor consumo de oxígeno, menor capacidad respiratoria, aumento del trabajo cardiaco y la menor inmunidad mediada por células ${ }^{(14)}$.

De acuerdo al reporte de los CDC, las embarazadas con COVID-19 tuvieron 3 veces más posibilidades de ingresar a terapia intensiva y recibir asistencia respiratoria mecánica, que la población de mujeres no embarazadas con similar condición de riesgo como presencia de comorbilidad, edad, raza y síntomas $^{(11)}$. En el presente estudio las 3 muertes maternas fueron debidas a complicaciones relacionadas al COVID-19, con 1 mortinato y una muerte neonatal. Ninguna de las fallecidas presentó comorbilidad. Las 2 comorbilidades maternas más frecuentes fueron la preeclampsia y la diabetes. Según estadísticas a nivel país durante el año 2019, los trastornos asociados a la hipertensión fueron del 21,4 por 100.000 habitantes $^{(13)}$.

En un reporte de casos de China el porcentaje de complicaciones de las mujeres con COVID-19 fue del $45 \%$ y el ingreso a la unidad de cuidados intensivos del $20 \%$, mientras que el $6 \%$ de los neonatos fueron hospitalizados ${ }^{(15)}$. Las cifras maternas fueron muy superiores a la encontrada en el presente estudio. Sin embargo, el porcentaje de neonatos hospitalizados fue casi 4 veces mayor que el reportado en dicho estudio. Las autoras atribuyen al elevado porcentaje de pretérminos, que, aunque la mayoría eran prematuros límites, con buen peso de nacimiento, se 
hospitalizaron para monitorizar la evolución por el riesgo que representaba la condición de la madre.

De los fallecidos, 2 podrían atribuirse al cuadro de la madre, pero con resultados de test negativo en ambos recién nacidos, aunque no se puede descartar la afectación perinatal, porque no se realizaron otros estudios además del hisopado. Las otras dos muertes no se relacionaron con el SARS-CoV-2. Los 3 neonatos con test positivo tuvieron síntomas leves y muy buena evolución.

Estos datos son similares a lo encontrado por Farghaly hasta la semana, et al, en una revisión retrospectiva, y seguimiento hasta los 14 días, algunos neonatos presentaron síntomas de COVID $19^{(16)}$.

La Academia Americana de Pediatría (AAP) recomienda-la alimentación con pecho materno a los hijos de madres positivas para SARS-CoV- $2^{(17)}$. Acorde con dichas recomendaciones, en este reporte, un elevado porcentaje de neonatos se alimentó con leche materna, tomando todas las precauciones sanitarias. Recientemente un estudio de cohortes con escaso número de participantes, que incluyó mujeres tanto positivas como negativas que amantaban a sus recién nacidos, realizó la búsqueda del SARS-CoV-2 en la leche materna. En una de las 18 muestras, el resultado fue positivo por PCR en tiempo real, pero el hisopado del recién nacido fue negativo y evolucionó sin síntomas ${ }^{(18)}$. Es posible que la positividad en la leche materna no implique infectividad.

En relación con la transmisión vertical, aunque se ha detectado alteraciones de la placenta en mujeres con COVID-19, no hay confirmación de hallazgo del virus en el feto, hasta la fecha ${ }^{(19,20)}$. Aunque las embarazadas por su condición no han participado de estudios clínicos, las vacunas de las plataformas mRNA fueron autorizadas. La respuesta inmunogénica a estas vacunas recientemente evaluada en 2 estudios, aunque con escaso número de pacientes, demostró pocos efectos adversos y el paso de los anticuerpos post vacuna a la leche materna y en el cordón umbilical, sugiriendo la posibilidad de efecto protector para el neonato de la infección por SARS$\mathrm{CoV}-2^{(21,22)}$.

El presente estudio tiene las limitaciones de ser una revisión retrospectiva, pero los datos de la historia clínica fueron corroborados por entrevista telefónica con las madres o sus familiares cercanos. El número de pacientes reclutadas puede que no haya sido suficiente para detectar otras condiciones relacionadas al SARS-CoV-2. Es pertinente seguir reportando las características de las embarazadas con COVID-19, para corroborar los hallazgos, fundamentalmente el elevado porcentaje de cesárea y de neonatos de pretérmino y el impacto de la vacunación masiva a embarazadas que acaba de implementarse en Paraguay.

\section{CONCLUSIÓN}

El 15,5\% de las embarazadas positivas ingresaron con formas moderadas a graves de COVID 19, de las cuales el 10,3\% ingresó a terapia intensiva y 3 fallecieron. La preeclampsia fue la complicación más frecuente. El porcentaje de cesárea, parto prematuro y hospitalización neonatal fueron muy elevados. Hubo 2 mortinatos y el 2,9\% de los nacidos vivos fallecieron. El hisopado nasofaríngeo neonatal, fue positivo en el $2,2 \%$, y tuvieron buena evolución clínica. El 78,1\% de los recién nacidos recibieron leche materna.

\section{CONTRIBUCIÓN DE AUTORES}

Sara Pereira Ferreira: Redacción del protocolo, recolección de los datos Redacción del manuscrito.

Dina Carrera: Redacción del protocolo, recolección de datos, revisión del manuscrito.

Mirta Mesquita: Concepción del tema, redacción del protocolo, análisis de los datos, corrección final del manuscrito. 


\section{REFERENCIAS}

1. Zhang L, Liu Y. Potential interventions for novel coronavirus in China: A systematic review. J Med Virol. 2020; 92(5):479-490. doi: https://doi.org/10.1002/jmv.25707

2. Carlos WG, Dela Cruz CS, Cao B, Pasnick S, Jamil S. Novel Wuhan (2019-nCoV) Coronavirus. Am J Respir Crit Care Med. 2020; 201(4):P7-P8. doi: https://doi.org/10.1 164/rccm.2014P7

3. Figueiro-Filho E, Yudin M, Farine D. COVID-19 during pregnancy: an overview of maternal characteristics, clinical symptoms, maternal and neonatal outcomes of 10,996 cases described in 15 countries. J Perinat Med 2020; 48(9):900-911. doi: https://doi.org/10.1515/jpm-2020-0364

4. Dubey P, Thakur B, Reddy S, Martinez CA, Nurunnabi M, Manuel SL, et al. Current trends and geographical differences in therapeutic profile and outcomes of COVID-19 among pregnant women - a systematic review and meta-analysis. BMC Pregnancy Childbirth. 2021; 21(1):247. doi: https://doi.org/10.1186/s12884-021-03685-w

5. Sola A, Rodríguez S, Cardetti M, Dávila C. COVID-19 perinatal en América Latina. Rev Panam Salud Publica. 2020;44e:47. doi: https://doi.org/10.26633/RPSP.2020.47

6. Villar J, Ariff S, Gunier RB, Thiruvengadam R, Rauch S, Kholin A, et al. The INTERCOVID Multinational Cohort Study. JAMA Pediatr. 2021; 175(8):817-826. doi: https://doi.org/10.1001/jamapediatrics.2021.1050

7. Moore KM, Suthar MS. Comprehensive analysis of COVID-19 during pregnancy. Biochem Biophys Res C om mun. 2021; 538:180-186. d o i : https://doi.org/10.1016/j.bbrc.2020.12.064

8. Ministerio de Salud Pública y bienestar Social. Protocolo para COVID 19: guía elmanejo de mujeres embarazadas y eventos obstétricos. [Internet] Versión II. 2021; [Consultado 2021 oct 20] Disponible en: https://www.mspbs.gov.py/ dependencias/portal/adjunto/24afa4-PROTOCOLOPARA COVID19GUIAPARAELMANEJODEMUJERESEMBAR AZADASYEVENTOSOBSTETRICOS.pdf

9. Zaigham M, Andersson O. Maternal and perinatal outcomes with COVID-19: A systematic review of 108 pregnancies. Acta Obstet Gynecol Scand. 2020; 99(7):823829. doi: https://doi.org/10.1111/aogs.13867

10. Dubey P, Reddy SY, Manuel S, Dwivedi AK. Maternal and neonatal characteristics and outcomes among COVID-19 infected women: An updated systematic review and meta-analysis. Eur J Obstet Gynecol Reprod Biol. 2020; 252:490-501. doi: https://doi.org/10.1016/j.ejo grb.2020.07.034
11. Zambrano L, Ellington S, Strid P, Galang RR, Oduyebo T, Tong VT. Update: Characteristics of Symptomatic Women of Reproductive Age with Laboratory-Confirmed SARS-CoV-2 Infection by Pregnancy Status - United States, January 22-October 3, 2020. MMWR. 2020; 69(44): 1641-47

12. Antoun L, Tawee NE, Ahmed Patni S, Honest H. Maternal COVID-19 infection, clinical characteristics, pregnancy, and neonatal outcome: A prospective cohort study. Eur J Obstet Gynecol Reprod Biol. 2020; 252:559562. doi: https://doi.org/10.1016/j.ejogrb.2020.07.008

13. Ministerio de Salud Publica y Bienestar Social. Subsistema de Información de las Estadísticas Vitales (SSIEV) Indicadores Básicos de Salud Paraguay 2019. [Internet] 2019; [Consultado 2021 oct 20] Disponible en:

14. Vlachodimitropoulou Koumoutsea E, Vivanti AJ, Shehata N, Benachi A, Le Gouez A, Desconclois C, et al. COVID-19 and acute coagulopathy in pregnancy. J Thromb Haemost. 2020; 18(7):1648-1652. doi: https://doi.org/10.1111/jth.14856

15. Gao YJ, Ye L, Zhang JS, Yin YX, Liu M, Yu HB, et al. Clinical features and outcomes of pregnant women with COVID-19: a systematic review and meta-analysis. BMC Infect Dis. 2020; 20(1):564. doi: https://doi.org/10.1186 /s12879-020-05274-2

16. Farghaly MAA, Kupferman F, Castillo F, Kim RM. Characteristics of Newborns Born to SARS-CoV-2-Positive Mothers: A Retrospective Cohort Study. Am J Perinatol. 2020; 37(13):1310-1316. doi: https://doi.org/10.1055/s-00401715862

17. American Academy of Pediatrics (AAP) Breastfeeding guidance post hospital discharge for mothers or infants with suspected or confirmes SARS- CoV-2 infecction. [Internet] 2019; [Consultado 2021 oct 20] disponible en https://services.aap.org/en/pages/2019-novel-coronaviruscovid-19-infections/clinical-guidance/breastfeedingguidance-post-hospital-discharge/

18. Kunjumon B, Wachtel EV, Lumba R, Remon J, Louie M, Verma M. et al. Breast Milk and Breastfeeding of Infants Born to SARS-CoV-2 Positive Mothers: A Prospective Observational Cohort Study. Am J Perinatol. 2021; 38(11):1209-1216. doi: https://doi.org/10.1055/s-0041-1731451

19. Vivanti AJ, Vauloup-Fellous C, Prevot S, Zupan V, Suffee C, Do Cao J. et al. Transplacental transmission of SARS-CoV-2 infection. Nat Commun. 2020; 11:3572 doi: https://doi.org/10.1038/s41467-020-17436-6

20. Schwartz DA. An Analysis of 38 Pregnant Women With COVID-19, Their Newborn Infants, and Maternal-Fetal 